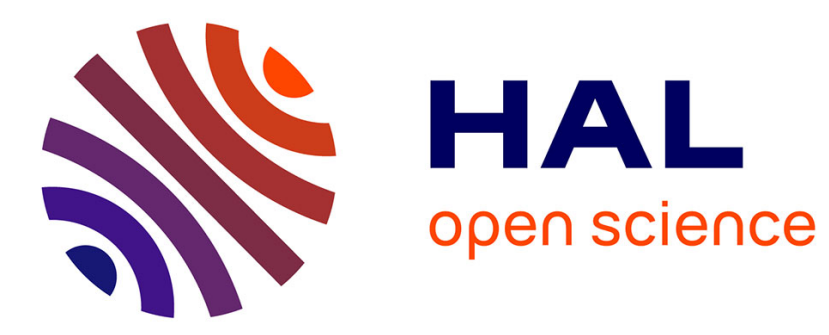

\title{
Hybride Hélène: figures du nom et contamination des genres chez Ronsard
}

Christophe Ippolito

\section{To cite this version:}

Christophe Ippolito. Hybride Hélène: figures du nom et contamination des genres chez Ronsard. Jeanne Garane. Hybrid Genres / L'Hybridité des genres, Brill., pp.106-122., 2018, French Literature Series, 10.1163/9789004361065_009. hal-03135445

\section{HAL Id: hal-03135445 \\ https://hal.science/hal-03135445}

Submitted on 24 Feb 2021

HAL is a multi-disciplinary open access archive for the deposit and dissemination of scientific research documents, whether they are published or not. The documents may come from teaching and research institutions in France or abroad, or from public or private research centers.
L'archive ouverte pluridisciplinaire HAL, est destinée au dépôt et à la diffusion de documents scientifiques de niveau recherche, publiés ou non, émanant des établissements d'enseignement et de recherche français ou étrangers, des laboratoires publics ou privés. 


\section{Hybride Hélène : figures du nom et contamination des genres chez Ronsard}

Version préparatoire de : Christophe Ippolito, "Hybride Hélène: figures du nom et contamination des genres chez Ronsard." Hybrid Genres / L'Hybridité des genres. Ed. Jeanne Garane. Amsterdam: Brill, 2018. 106-122.

\section{Hybride Hélène : figures du nom et contamination des genres chez Ronsard}

\section{Résumé}

Dans les années 1570, Ronsard, éclipsé en tant que poète lyrique par la renommée de Desportes, était surtout renommé pour ses écrits épiques et sa poésie scientifique, soit pour des œuvres telles que les Hymnes et La Franciade. En choisissant le nom d'Hélène dans les Sonnets pour Hélène, Ronsard « imite » un modèle célèbre, tente de fragiliser la réputation de Desportes, veut regagner son rang auprès du public. Le nom d'Hélène évoque Hélène de Surgères, supposée être son modèle et l'objet de sa cour poétique, il nous rappelle aussi l'héroïne épique d'Homère. Mais en fait, la principale préoccupation de Ronsard semble être moins Hélène de Surgères que l'image que le nom de celle-ci génère, nom signe et vecteur d'hybridité. Ronsard joue sur les vraies et fausses étymologies, mais aussi sur d'autres associations. Le couple res / verba (Hélène / étymologies), au lieu d'indiquer une dualité rassurante dans laquelle les mots « reflètent » la réalité, affirme par ailleurs l'hybridité-duplicité constitutive du discours de la séduction... et de la poésie lyrique. Et d'autre part, la réécriture du mythe d'Hélène passe par une contamination inter-générique. Tout le monde connaît le mythe raconté par Homère dans l'Iliade, mais Euripide développe dans sa pièce Hélène une version tout-à-fait différente du mythe, version avec laquelle Ronsard joue. Dans cette pièce, Hélène ne va pas à Troie : c'est une fausse Hélène qui s'y rend. Comme Euripide, Ronsard a écrit à une époque où la guerre faisait rage, de sorte que l'utilisation de cette version du mythe pourrait bien être une tentative d'intégrer aux jeux du désir un plaidoyer contre la guerre. En dernière analyse, le caractère hybride de l'œuvre bloque toute prétention à une interprétation unique ou unifiée du cycle.

\section{Abstract}

In the 1570 s, Ronsard, eclipsed by Desportes' fame as a writer of love sonnets, was known above all for his epic and scientific poetry, i.e. for works such as the Hymns and the Franciade. In a letter to Monsieur de Sainte-Marthe, Ronsard shows his disdain for "petits et menus fatras come elegies, epigrames et sonnetz," and in the Preface sur la Franciade, he celebrates the glory of the epic poet. By choosing the name of Helen in Sonnets pour Hélène, Ronsard "imitates" a famous model (Homer) and throws darts against Desportes' reputation. Ronsard's primary concern seems to be less Hélène de Surgères than the image her name generates, and Homer's epic heroin. In the sonnets, the couple res/verba (Hélène/etymologies), instead of indicating a reassuring duality in which words "reflect" reality, asserts the essential uncertainty (or duplicity) constitutive of lovers' discourses... and lyric poetry. The rewriting of Helen's myth is yet another way of expressing this ambivalence. Everyone knows the myth of Helen as told by Homer, but Euripides, after Herodotus or Stesichorus, develops in Helen a quite dissimilar 
version of the myth, a version Ronsard playfully integrates into his sonnets. No love story here: this might be a way to condemn war, raging in France at this time, while celebrating the pleasure of the text. In the end, there cannot be a unified interpretation of this hybrid text.

Comme Terence Cave le dit, « les problèmes d'écriture et de lecture posés par la poésie de Ronsard peuvent, dans les termes les plus généraux, être attribués à sa prolifération ${ }^{1}$ »; ainsi, continue-t-il, « le texte et son lecteur sont pris dans les nœuds d'une poursuite de l'intégration qui, sinon inutile, n'en est pas moins condamnée à l'échec » (Cave 1997, 243). Afin de surmonter cette résistance du texte, poursuit-il, « la possibilité subsiste, cependant, de faire fructifier cette difficulté en l'exploitant comme un thème critique » (Cave 1997, 243). Dans cette optique il est donc approprié de souligner la récurrence de la non-intégration comme un aspect majeur des Sonnets pour Hélène. Or comme il arrive paradoxalement, cette non-intégration n'est nulle part plus évidente que dans le nom même de l'être aimé. Paradoxalement en effet, les Sonnets pour Hélène montrent un cratylisme renouvelé ${ }^{2}$. Une relation est ainsi suggérée entre verba et res. Mais ce même cratylisme conduit à l'illusion, si l'on admet que les mots ne sont que des images des choses, et qu'en fait, toute image est en soi insaisissable.

Dans les Sonnets pour Hélène 3 , en II, 6, dans un sonnet intitulé « Anagramme », Ronsard célèbre la puissance poétique du nom d'Hélène :

Les noms ont efficace \& puissance \& vertu :

Je le voy par le tien lequel m'a combatu [...]. (Sonnets pour Hélène 109, v. 9-10)

Cette opinion sur les vertus poétiques peut être attribuée à Platon, ce qu'une variante montre :

Les noms (ce dit Platon) ont tresgrande vertu [...]. (Sonnets pour Hélène 109, v. 9, variante)

Plusieurs sonnets dans ce recueil de poèmes célèbrent le fameux nom de la bien-aimée, comme c'était le cas dans Sonnets à Cassandre. Tel est le cas dans le poème ci-après :

Heureux celuy qui souffre une amoureuse peine

Pour un nom si fatal [...]. (Sonnets pour Hélène 32 ; I, 3 v. 5-6)

Nul doute que Ronsard tente ici de répondre aux exigences de sa réputation non usurpée de « Homère françois ». Dans les années 1570, Ronsard, éclipsé en tant que poète lyrique par la

\footnotetext{
${ }^{1}$ Terence Cave, Cornucopia. Figures de l'abondance au XVIe siècle : Érasme, Rabelais, Ronsard, Montaigne, trad. Ginette Morel, Paris, Macula, coll. «Argô », 1997, 243. La version originale est la suivante : The Cornucopian Text: Problems of Writing in the French Renaissance, Oxford, Clarendon Press, 1979. La version française comporte ce que Cave appelle des « retouches » et des « raccourcis » (5). Les deux versions (notées 1979, 1997) seront utilisées. Pour mieux apprécier la réception du livre de Cave, notamment en France, au-delà des polémiques inutiles, le lecteur se reportera avec profit à la «Préface de l'édition française » (5-14).

${ }^{2}$ Sur le nom d'Hélène ( "Hélène”, ou le cratylisme déclaré », 217-227), comme sur d'autres noms de « femmes aimées » chez Ronsard et d'autres poètes de la période, voir François Rigolot.

${ }^{3}$ Pierre de Ronsard, Sonnets pour Hélène (1578), éd. Malcolm Smith, postface de Daniel Ménager, Genève, Droz, coll. « Textes Littéraires Français » [T.L.F.], no 167, 1998 [1 ${ }^{\text {ère }}$ édition, 1970]. Le Premier Livre des sonnets d'Hélène est ci-après noté I, Le Second Livre des sonnets pour Hélène est ci-après noté II.
} 
renommée de Desportes ${ }^{4}$, était surtout connu pour ses écrits épiques et sa poésie scientifique, soit pour des œuvres telles que La Franciade et les Hymnes (œuvres d'ailleurs majeures). Dans une lettre à M. de Sainte-Marthe, il semble montrer un certain mépris pour les « petits et menus fatras come elegies, epigrames et sonnetz » où les poètes « ne gardent ny ordre ny temps ${ }^{5}$ ». Le nom d'Hélène évoque Hélène de Surgères, la jeune suivante de Catherine de Médicis qui est supposée être son modèle et l'objet de sa cour poétique, il nous rappelle aussi l'héroïne épique d'Homère (et Homère reste un modèle indépassable pour Ronsard et ses contemporains ${ }^{6}$ ). En choisissant stratégiquement le nom d'Hélène, Ronsard vieillissant fait au moins deux ou trois choses : il « imite » un modèle célèbre, et tente de fragiliser la réputation de Desportes et de reconquérir son aura de poète lyrique (et une partie de son « public », à la cour notamment). Cela est clair dans le sonnet II, 54 où Ortygie (une synecdoque pour Diane et un autre nom pour l'île de Délos, où Diane serait née), évoque l'antagoniste Desportes à travers son célèbre ouvrage Amours de Diane :

Je te voulais nommer pour Helene, Ortygie

Renouvellant en toy d'Ortyge le renom.

Le tien est plus fatal : Helene est un beau nom,

Helene, honneur des Grecs, la terreur de Phrygie :

Si pour sujet fertil Homere t'a choisie,

Je puis suivant son train qui va sans compagnon,

Te chantant m' honorer, \& non pas toy, sinon

Qu'il te plaise estimer ma rude Poesie. (Sonnets pour Hélène 162 ; II, 54, vv. 1-8)

Les sonnets sont ainsi une façon pour Ronsard de « [s']honorer », de se célébrer soi-même, comme poète lyrique et sans doute aussi comme poète de cour ; son nom apparaît au moins dans trois sonnets (respectivement II, 2, 12, 56) :

[...] La parfaite amitié que Ronsard vous portoit, [...]. (Sonnets pour Hélène 105, v. 2)

[...] Je vous aime, Ronsard, par seule destinee, [...]. (Sonnets pour Hélène 116, v. 3)

[...] De moy seule, Ronsard, l'escrivain vous serez. (Sonnets pour Hélène 164, v. 4)

La prolifération du nom illustre ajoute à la célébrité du poète et à la mise en abyme généralisée, le cycle se tournant vers l'intérieur de lui-même. En fait, la principale préoccupation de Ronsard semble être moins Hélène de Surgères que l'image que le prénom de celle-ci génère. D'ailleurs, de nombreux sonnets finalement publiés dans le cycle ont été détournés des Amours Diverses. Le Sonnet I, 16, selon Claude Binet, biographe de Ronsard au seizième siècle, était d'abord destiné à une autre femme, Diane de Cossé-Brissac selon certains, mais selon Malcolm Smith Jane de Brissac, associée avec Hélène de Surgères dans un sonnet de Baïf (c $f$. Sonnets pour Hélène 49, note 1), ce qui nie l'unité présupposée du cycle que certains critiques furent autrefois prompts à attribuer à une seule femme.

\footnotetext{
${ }^{4}$ Voir sur ce point les travaux de Claude Faisant et de Richard A. Katz.

${ }^{5}$ Pierre de Ronsard, La Franciade, Euvres complètes, éd. Paul Laumonier et al., tome XVI, 24. Cf. aussi Malcolm Smith, qui cite la Preface [sic] sur la Franciade où Ronsard célèbre le poète épique « aux depens du simple poete d'amour » (Sonnets pour Hélène 8, Introduction).

${ }^{6}$ Albert Py a pu tirer cet ensemble de sonnets et le « je » poétique qui les anime du côté de l'autobiographie. Ce n'est pas notre objectif ici.
} 
Il nous semble que le choix de Surgères s'imposait : son nom ouvrait la possibilité de l'anagramme ; si [H]élène de Surgères est «LE RÉ DES GENEREUS » (Sonnets pour Hélène 109 ; II, 6, v. 14), alors l'amant est « généreux » lui-même, comme poète inspiré et célébré, et surtout comme homme de noble condition, ce que Desportes n'était pas. La prolifération du nom propre, en bloquant toute interprétation « unifiée », met en danger le processus d'attribution d'une signification précise à ce cycle de poèmes d'amour, ce qui peut être facilement compris en termes aristotéliciens :

En effet, ne pas signifier une chose unique, c'est ne rien signifier du tout, et si les noms ne signifiaient rien, on ruinerait tout échange de pensée entre les hommes, et, en vérité, aussi avec soi-même ; car on ne peut pas penser si on ne pense pas une chose unique; et, si on le peut, un seul nom pourra être assigné à cette chose. (Aristote, Métaphysique 96 [Gamma, 1006 b])

Cette prolifération se trouve d'abord dans des figures de style impliquant des jeux de mots : antonomase et annominatio. Par exemple, dans le sonnet I, 3, le narrateur définit la bien-aimée comme le souffle de sa vie (une sorte de pneumos ou de zoё donc, un principe organique de vie qui trouverait sa réalisation dans le bios de Ronsard), ce qui est tout à fait conforme « avec la philosophie des hymnes saisonniers » (Stone 167 ; ma traduction) :

Ma douce Helene, non, mais ma bien douce haleine, Qui froide rafraischis la chaleur de mon coeur, Je prens de ta vertu cognoissance \& vigueur, [...]. (Sonnets pour Hélène 32, v. 1-3)

Pour un lecteur du XVI ${ }^{\mathrm{e}}$ siècle, le mot « haleine » va sans aucun doute de pair avec l'idée d'inspiration, comme le souligne Malcolm Smith, citant une préface à un cycle de poèmes d'amour de Guillaume de La Tayssonnière, cycle intitulé « La Sourdine » :

Ronsard mieux halené pourra tandis sonner [...]. (Sonnets pour Hélène 10, Introduction)

Ailleurs dans les Sonnets pour Hélène, le narrateur est représenté comme étant « nourri » par la bien-aimée, non seulement comme amant, mais surtout comme poète : « [...] Helene [sic] est tout mon cœur, mon sang \& mes propos [...] » (Sonnets pour Hélène 87, I, 51, v. 3). En assimilant le souffle de sa vie (« cœur», « sang ») d'une part, avec son inspiration poétique ( «mes propos ») d'autre part, le narrateur semble célébrer en Hélène non seulement sa bienaimée, le récepteur, le telos, l'objet des sonnets, mais aussi l'outil, son inspiration poétique, bref, une manifestation de la furor poetica, telle qu'elle apparait dans le quatrain suivant :

Ne verse point de l'eau sur ma bouillante flame,

Il faut par ta douceur mes Muses enhardir :

Ne souffre de mon sang le bouillon refroidir,

Et tousjours de tes yeux aiguillonne moy l'ame. (Sonnets pour Hélène 96-97 ; I, 59, v. 5-8.)

L'annominatio est également utilisée par Ronsard comme une figure de copia, de prolifération. Le processus d'annominatio, " remotivation du nom propre par étymologie » selon la définition de Littré7, déploie une multiplicité de sens qu'on peut « extraire » poétiquement de la « mine » que constitue le nom d'Hélène :

\footnotetext{
${ }^{7}$ « Annomination : Remotivation du nom propre par étymologie, ou métanalyse ou traduction. En d'autres termes, un nom propre est utilisé avec le sens soit du nom commun soit des segments qui l'ont formé ou que l'on peut y déterminer, même par simple homophonie, voire dans une autre langue ».
} 
Ny la douce pitié, ny le pleur lamentable

Ne t'ont baillé ton nom : ton nom Grec vient d'oster,

De ravir, de tuer, de piller, d'emporter

Mon esprit \& mon coeur, ta proye miserable. (Sonnets pour Hélène 112-113 ; II, 9, v. 1-4.)

Ce faisant, le nom d'Hélène est signe et vecteur d'hybridité. Ronsard joue sur les vraies et fausses étymologies. Hélène est celle qui est « ravie », " prise », du verbe élein, et n'est pas associée à un « pleur lamentable » (éleeinon dakruon), voire à un " objet de pitié » (du verbe eleein), la pitié renvoyant à la tragédie, le pleur au pathos. Et il est d'autres associations, ainsi « hellène » pour « grecque ». Le couple res / verba (Hélène / étymologies), au lieu d'indiquer une dualité rassurante selon laquelle les mots " reflètent la réalité », affirme par ailleurs l'hybridité-duplicité essentielle et constitutive du discours de la séduction... et de la poésie lyrique. Comme le souligne Terence Cave,

Les utilisateurs de la langue sont dupés par des habitudes linguistiques profondément enracinées lorsqu'ils supposent que le discours reflète, représente, ou mime la réalité, et ont tendance à sous-estimer son inclinaison congénitale à déroger aux « choses » qu'il prétend représenter. Ou, pour dire la même chose sous un autre angle, les mots reconstituent les choses dans leur propre moule (lexical, syntaxique, phonologique), affirmant leur domination sur ce qu'ils étaient censés servir. Ainsi le langage est-il à la fois déviant (déviant de son « origine » dans la réalité) et sournois (cachant sa duplicité, sapant l'assurance avec laquelle il est couramment utilisé). La duplicité peut être partiellement masquée, mais jamais éliminée, au moyen d'une rhétorique réfléchissante qui exploite les dispositifs de jeux de mots, et en associant les opérations du langage avec des thèmes de plaisir. (Cave 1979, Introduction, xviii ; ma traduction)

Si nous citons de façon extensive le livre de Cave, c'est que parler de plaisir textuel semble particulièrement pertinent à la fois dans le contexte général de la Renaissance et dans le microcontexte des Sonnets pour Hélène, texte de plaisir aussi bien que texte de corne d'abondance. Et la façon dont Cave construit le détail de son argumentation nous semble fort efficace. Ce plaisir serait-il notamment dans l'abondance que l'annominatio sert si bien ? Dès les tercets du sonnet I, 3, la dame a deux postulations, Hélène et Pénélope :

Nom, malheur des Troyens, sujet de mon souci, Ma sage Penelope \& mon Helene aussi, Qui d'un soin amoureux tout le cœur m'enveloppe :

Nom, qui m'a jusqu'au ciel de la terre enlevé,

Qui eust jamais pensé que j'eusse retrouvé,

En une mesme Helene, une autre Penelope! (Sonnets pour Hélène 32 ; I, 3)

Chez Homère, Pénélope et Hélène forment un contraste parfait : en bref, Pénélope est

l'incarnation de la chasteté et la vertu, tandis qu'Hélène est exactement le contraire, ainsi que l'incarnation de la beauté. Cependant, chez Ronsard, il est impossible d'assimiler Pénélope avec une femme idéalisée à la manière néoplatonicienne, et de voir en Hélène une figure opposée à cette dernière. Ainsi, le sonnet I, 4 se consacre exclusivement à une équation platonicienne d'une maîtresse parfaite qui de par sa vertu chaste ressemble plus à Pénélope qu'à Hélène (Sonnets pour Hélène 33). À un moment du cycle, le poète-amant demande à la femme de changer son nom ([...] « change de nom Maistresse / Un autre plus cruel te convient beaucoup mieux » (Sonnets pour Hélène 177 ; II, 68, v. 13-14, et donc en fin de sonnet). De la même manière, il est par exemple impossible de comparer le poète-« amant» à Ulysse, ancien prétendant d'Hélène et époux de Pénélope, sinon peut-être sur le plan bien incertain de l'ingéniosité, que celle-ci se situe 
en acte ou en parole (poétique), et ce en limitant la « reconnaissance » du moi à celle, suffisante dans certaines doctrines, de ses qualités.

D'autre part, la réécriture du mythe d'Hélène est une façon d'opérer une amplificatio du nom de la dame, amplificatio qui passe par une contamination inter-générique. On le sait,

Examiner l'utilisation par Ronsard de l'imagerie mythologique revient à avoir un aperçu de son univers poétique dans son ensemble. Elle représente sous une forme quintessentielle sa pratique humaniste de «l'imitation », de l'adaptation de thèmes, d'images et de styles des poètes de l'Antiquité classique. (Cave 1973,159 ; ma traduction)

Tout le monde connaît le mythe raconté par Homère dans l'Iliade, mais Euripide, après Hérodote ou Stésichore, développe dans sa pièce Hélène une version tout-à-fait différente du mythe. De nombreuses allusions à la version d'Euripide existent chez Ronsard et confirment sa parfaite connaissance de cette œuvre, ainsi dans notre texte :

Laisse de Pharaon la terre Egyptienne [...]. (Sonnets pour Hélène 146 ; II, 40, v.1)

Il ne faut pas oublier que dans la seule élégie de ce cycle, le « narrateur » commentant sa vie, dit :

$[\ldots]$

Ayant toujours és mains pour me servir de guide

Aristote ou Platon, ou le docte Euripide. (Sonnets pour Hélène 188, v. 9-10 ; poème non numéroté).

Ailleurs, Ronsard fait encore directement allusion à la version d'Euripide, ce qui montre sa familiarité avec cette version ${ }^{8}$.

Dans l'Hélène d'Euripide, Hélène reste vertueuse. Dans cette pièce constellée de surprises et de renversements aussi merveilleux que soudains, et semée d'une ironie qui questionne sans cesse les grands textes homériques, elle est prise par Hermès et envoyée en Égypte - une Égypte qui dans la culture grecque d'alors évoque une certaine grandeur mais aussi des sortilèges, maléfices, et autres manifestations de l'irrationnel. C'est non pas Hélène mais une image de celle-ci fabriquée par une Héra jalouse - Pâris on le sait avait préféré Aphrodite à Héra et Athéna sous le rapport de la beauté - que Pâris emmène à Troie. Une image trompeuse, comme peuvent l'être les images. En Égypte, la «véritable » Hélène vit sous la protection du roi Proteus. À la mort de ce dernier, cette « véritable » Hélène est pressée par son fils Theoklymenos (Euripide joue sans doute ici sur le personnage d'Homère portant le même nom : autre exemple d'annominatio), mais Ménélas arrive alors à son tour en Égypte, avec la fausse Hélène. Vrai contre faux : la fausse Hélène (l'image) se révèle comme telle, comme illusion, et la guerre de Troie comme inutile ; cette version « pacifiste » ou tout au moins plus pacifique met en question la guerre en général. Et on en vient à douter, productivement d'ailleurs, de certaines choses, de certains héros, de certains dieux. La guerre de Troie a-t-elle eu lieu ? Qui est Hélène - ou tout au moins, que représente-t-elle ? À la fin de la pièce, il ne reste plus au « vrai » couple qu'à partir d'Égypte. On reconnaît facilement dans ce résumé certains aspects et fonctionnalités des sonnets, et surtout, ce que Michel Simonin a souligné, le lien entre le trompeur « songe amoureux » et l'eidolon:

\footnotetext{
${ }^{8}$ « [...] le plaisir du sage Roy Protée / Qui d’Helene retint la figure empruntée » (« Élégie », v. 29-30, in Le Premier livre des poèmes, in Pierre de Ronsard, Euvres complètes, éd. Jean Céard et al., tome II, 667 ; voir particulièrement 667, note 3, sur Hélène d'Euripide. Cf. aussi Dauvois 203-205.
} 
Le songe amoureux déceptif place souvent dans l'étreinte du narrateur « une forme douteuse », qui est à la fois le remède à son « ennuy » et la source de sa désillusion. Surgères héllénisée ne rend pas seulement « l'homme [...] malheureux » parce qu'il se « repaist d'un songe », elle fraternise elle-même, par son absence de sensualité, le silence de son corps, sa sympathie pour les morts ou encore son goût pour le platonisme avec le monde de l'eidolon dont elle procède ${ }^{9}$. (Simonin 129)

En fait, la thématique du « songe amoureux », du rêve, du fantôme trompeur, et de la désillusion anime le cycle. « Ce que nous avons ici est un territoire mouvant situé au point où la réalité et l'illusion, la vérité et la fiction, la vie et l'art convergent », écrit Cave (1973, 159 ; ma traduction). L'eidolon est condamnée, dans un sens platonicien, à être à la fois insatisfaisante et trompeuse :

L'autre amitié ressemble aux enfans sans raison :

C'est se feindre une flame, une vaine prison,

Où le feu contrefait ne rend qu'une fumée. (Sonnets pour Hélène 94 ; I, 57, v. 12-14)

«L'autre amitié » peut-elle être rapprochée de «l'amour céleste » qui « [...] pousse les ames guidées / Aux belles contemplations ${ }^{10} »$ ? Il reste que Ronsard comme on sait célèbre le corps :

En choisissant l'esprit vous estes mal-apprise,

Qui refusez le corps, à mon gré le meilleur :

De l'un en l'esprouvant on cognoist la valeur,

L'autre n'est rien que vent, que songe \& que feintise.

(Sonnets pour Hélène 197 ; Appendice, I, v. 1-4,)

On trouve des propos semblables en I, 50 :

Or vous aimez l'esprit, \& sans discrétion

Vous dites que des corps les amours sont pollues.

Tel dire n'est sinon qu'imagination

Qui embrasse le faux pour les choses cognues :

Et c'est renouveller la fable d'Ixion,

Qui se paissoit de vent \& n'aimoit que des nues. (Sonnets pour Hélène 85 ; I, 50, v. 9-14)

Le sonnet le plus platonicien de tous (II, 53) reflète cette situation. Tout comme selon la définition de Boccace la poésie ne révèle qu'une vérité voilée sous un manteau fabuleux, chez Ronsard, le manteau fabuleux de la mythologie voile l'essence d'Hélène, plus opaque que transparente :

Un bon-heur te defaut : c'est qu'en venant çà bas

Couverte de ton voile on ne t'admira pas,

Tant fut ton excellence à ce monde incognue,

Qui n'osa regarder les rayons de tes yeux :

Seul je les adoray comme un thresor des Cieux,

Te voyant en essence, \& les autres en nue. (Sonnets pour Hélène 161 ; II, 53, v. 9-14)

Dans la pièce d'Euripide, Hélène renvoie aux mythes sur elle-même sans beaucoup d'enthousiasme. Chez Ronsard, l'irrévérence du jeu sur les mythes peut servir à dénoncer les

\footnotetext{
${ }^{9}$ Deux notes indiquent les références de Simonin : « une forme douteuse » renvoie à Sonnets pour Hélène 147 (II, 41, v. 5),

«l'homme [...] malheureux » parce qu'il se « repaist d'un songe » à Sonnets pour Hélène 187 (II, 74, v. 14).

${ }^{10}$ Pierre de Ronsard, Ode au Roy Charles luy donnant un Leon Hebrieu, Les Estoilles, Euvres complètes, éd. Paul Laumonier et al., tome XVII, Première partie, 62, v. 13-14.
} 
illusions des humains, et particulièrement des amoureux. Cependant, comme Euripide, Ronsard a écrit à une époque où la guerre faisait rage, de sorte que l'utilisation de cette version du mythe pourrait bien être non seulement une tentative de "blanchir" Hélène, mais aussi d'intégrer aux jeux du désir un plaidoyer contre la guerre. Le désir comme la guerre seraient causés par des erreurs, des illusions entretenues. Chez Euripide, le chœur des femmes grecques prisonnières dénonce aussi bien le désir que la guerre. Et si les Sonnets pour Hélène ne sont pas une critique si voilée que ce soit du contenu de l'Iliade, il semble bien que la guerre soit critiquée dans le cycle. Un sonnet publié dans les Sonnets pour Hélène en 1584 et dans les Amours Diverses en 1578 et 1587 vient renforcer cette interprétation :

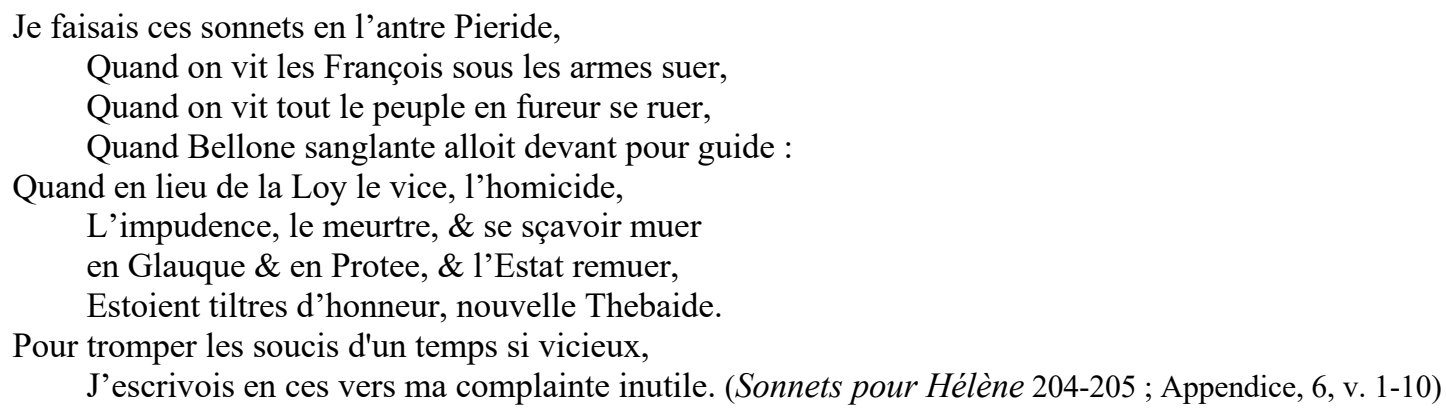

Même idée en I, 51 :

[...] Qu'il nous donne la paix, \& de tes bras l'enserre. (Sonnets pour Hélène 87 ; I, 51, v. 11)

Et en II, 26 :

Au milieu de la guerre, en un siecle sans foy, Entre mille procez, est-ce pas grand' folie

D'escrire de l'Amour ? [...]. (Sonnets pour Hélène 129; II, 26, v. 1-3)

En fait, il n'y a pas d'unité dans ce cycle. L'unité présupposée de la scripta est manifestement divisée par la prolifération du nom d'Hélène, autant que par les différentes voix qui célèbrent ces instances du nom. Car la voix poétique ne tend nulle part à une unité rassurante. En effet, le poète met en scène maintes voix contradictoires qui interfèrent les unes avec les autres. Il y a les voix de Platon et de Pétrarque ainsi de que leurs « adversaires », et on pourrait facilement montrer leur présence dans le cycle, dans le détail. Cela ne surprend pas, si seulement nous pensons avec Cave que « selon Érasme, l'écrivain doit affirmer son indépendance à la fois par la multiplication et la fragmentation de ses modèles » (1979 xi, Introduction; ma traduction). Et nous retrouvons ce processus à propos des différents modèles mythiques commentés. L'imitation des mythes cède la place à l'interprétation et au commentaire, et les différentes incarnations d'Hélène bloquent toute prétention à une interprétation unique ou unifiée du cycle de sonnets.

Dans son écriture, le poète qui choisit d'examiner différentes interprétations tente peut-être de trouver un équilibre entre ses différents choix. Ce faisant, le poète agirait presque comme l'amant sage, platonicien ou autre, qui doit d'abord « régler » sa volonté afin de se débarrasser de l'amour en tant que (mauvaise) passion induisant en erreur, et de privilégier un désir plus modéré et contrôlé. Dans cette perspective, le désir semble plus généreux que l'amour, dans la mesure où il est censé être ouvert à toutes les beautés (ou, dit autrement, donner une chance à chaque forme de beauté). Au-delà, le désir sans amour semble le sentiment idéal pour un anti-idéalisme qui 
s'opposerait à la quête d'une essence inaccessible. Le désir sage appelle simplement à une recherche de la beauté dans ses manifestations. Dans le même temps, jouer sur différentes incarnations d'Hélène est une façon de reconnaître le fait que la bien-aimée est inconnaissable ; si la seule façon de connaître absolument quelqu'un est de savoir tout ce qu'il ou elle désire, le désir de l'être aimé ne peut pas être entièrement connu. Cette " opacité » de la bien-aimée doit être acceptée par l'amant. Le désir est célébré dans le sonnet I, 56 :

\author{
Le desir au desir d'un nœud ferme lié \\ Par le temps ne s'oublie \& n'est point oublié, \\ Il est tousjours son tout, contenté de soy-mesme. (Sonnets pour Hélène 94 ; v. 6-8)
}

Ainsi, les Sonnets pour Hélène célébreraient le désir plus que l'amour, comme en témoignent les sonnets de la fin du recueil. Le cycle se termine par le fameux verset :

Car l'Amour \& la Mort n'est qu'une mesme chose [...]. (Sonnets pour Hélène 195 ; II, 79, v. 14)

Ce verset développe un topos : l'amour de la créature, selon la perspective augustinienne, conduit à l'illusion et à la mort. En outre, ce qui semble être une dernière note de pétrarquisme dans ce concetto est une façon, si l'on considère comme Nancy J. Vickers que la poésie pétrarquiste est sujette à la pétrification, de reconnaître que cette pétrification est symbolique de la mort, et que seul un poète détaché de ces sortes de topoi pourrait réussir à prétendre être, selon les normes de son temps, sur le chemin de l'immortalité.

Ainsi nous avons vu que la principale préoccupation de Ronsard semble être de se célébrer lui-même comme poète au mieux de ses moyens, maîtrisant toutes les potentialités du jeu poétique (soulignons en particulier la façon subtile dont les thèmes et figures du double, de la dualité, ou de la duplicité, envahissent le cycle). La dualité se manifeste à bien des égards, le cycle étant fondé sur une variété presque sans fin de couples possibles : Hélène / Pénélope, Hélène / étymologies prétendues ou homophonies, Hélène / Ortygie, Euripide / Homère, amour / désir. En fin de compte, comme il est montré à travers le jeu sur le nom de la dame, le poète« amant » est poète plus qu' « amant » : l'amant n'est que représentation. Ce qui n'interdit pas, en même temps, de remettre par ailleurs en question une certaine idée assez répandue de l'amour (au profit d'un désir plus détaché, distancié et joueur). Et même le désir que Ronsard célèbre ici est à la fois l'objet d'une dépense et le lieu d'une perte, voire d'une illusion inévitable :

\title{
[...] Tu te vas decevant [...]. (Sonnets pour Hélène, I, 25, v. 9, p. 58)
}

Un mouvement de déception et de désillusion sape le statut (représentationnel) de l' «amant » en élevant celui du poète, simultanément. Le poète pourrait bien célébrer ici le plaisir du texte, sinon un autre type de plaisir :

Encor que je me trompe, abusé du contraire

Pour me faire plaisir, Helene, je le croy. (Sonnets pour Hélène 59 ; I, 26, v. 13-14)

En fait, le caractère hybride de l'œuvre (manifeste dans les différentes incarnations d'Hélène) semble bloquer toute prétention à une unité strictement interne (et définitive) du cycle et de la constellation formés par les sonnets, en les ouvrant à un éventail quasiment illimité de lectures et de questions ou « réponses » potentielles (contextuelles, intratextuelles, intertextuelles, idéologiques, esthétiques, éthiques, politiques...), qui s'entrelacent les unes avec les autres : 
dans ce texte ouvert s'il en est, l'hybridité est éminemment productrice. Comme le texte, destinateur et destinataires sortent gagnants de ce jeu-là.

Christophe Ippolito

Georgia Institute of Technology \& Écritures (EA 3943)

\section{Ouvrages cités}

Aristote, Métaphysique [ePub, PDF]. Trad. J. Tricot (1953). Paris : Éditions Les Échos du Maquis, 2014.

Cave, Terence. « Ronsard's mythological universe ». Ronsard the poet. Éd. Terence Cave. London : Methuen, 1973. 159-208 [chapitre IV].

Cave, Terence. The Cornucopian Text. Problems of Writing in the French Renaissance. Oxford : Clarendon Press, 1979.

Cave, Terence. Cornucopia. Figures de l'abondance au XVI siècle : Érasme, Rabelais, Ronsard, Montaigne. Trad. Ginette Morel. Paris : Macula, coll. « Argô », 1997.

Dauvois, Nathalie. Mnémosyne : Ronsard, une poétique de la mémoire. Paris : Champion, 1992.

Faisant, Claude. « Les relations de Ronsard et de Desportes ». Bibliothèque d'Humanisme et de Renaissance, XXVIII, 1966. 323-353.

Katz, Richard A. Ronsard's French Critics, 1585-1828. Genève : Droz, coll. " Travaux d'Humanisme et de Renaissance » [THR], 1966.

Py, Albert. « Les séquences autobiographiques dans Ronsard » [Chapitre Premier], Imitation et Renaissance dans la poésie de Ronsard. Genève : Droz, 1984. 31-69.

Rigolot, François. Poétique et onomastique : l'exemple de la Renaissance, Genève, Droz, coll. « Histoire des idées et critique littéraire », nº 160, 1977.

Ronsard, Pierre (de), Sonnets pour Hélène (1578). Éd. Malcolm Smith. Genève : Droz, coll. «Textes Littéraires Français » [T.L.F.], nº 167, 1998 [1 ère édition, 1970].

. Euvres complètes [20 tomes]. Édition critique par Paul Laumonier, révisée et complétée

par I. Silver et R. Lebègue. Paris : Librairie Marcel Didier, Société des textes français modernes (S.T.F.M.), 1914-1975. 
Le Premier livre des poèmes. Euvres complètes. Éd. Jean Céard, Daniel Ménager et Michel Simonin. Paris : Gallimard, coll. « Bibliothèque de la Pléiade », 1994, tome II.

Simonin, Michel. « Hélène avant Surgères : Pour une lecture humaniste des Sonnets pour Hélène ». Sur des vers de Ronsard (1585-1985). Actes du colloque international (Duke University). Éd. Marcel Tetel. Paris : Aux Amateurs de livres, 1990. 127-143.

Stone, Donald, Jr., Ronsard's Sonnet Cycles : A Study in Tone and Vision. New Haven and London : Yale U.P., Yale Romanic Studies, Second Series 14, 1966.

Vickers, Nancy J., « Les Métamorphoses de la Méduse : Pétrarquisme et pétrification chez Ronsard ». Sur des vers de Ronsard (1585-1985). Actes du colloque international à Duke University. Éd. Marcel Tetel. Paris : Aux Amateurs de livres, 1990. 159-170. 